\title{
The Formation of Polypyrrole at Iron from
1-Butyl-3-methylimidazolium Hexafluorophosphate
}

\author{
Anna M. Fenelon* and Carmel B. Breslin*,z \\ Department of Chemistry, National University of Ireland Maynooth, Maynooth, County Kildare, Ireland
}

\begin{abstract}
The ionic liquid, 1-butyl-3-methylimidazolium hexafluorophosphate, was used to deposit adherent and highly conducting polypyrrole coatings at pure iron using a simple potentiostatic technique. Adherent and highly conducting polypyrrole films were formed at the iron substrate using potentials in the range from 1.0 to $1.4 \mathrm{~V}$ and with pyrrole concentrations between 0.1 and 0.3 mol dm${ }^{-3}$. An increase in the applied potential, or the concentration of the monomer, gave rise to an increase in the rate of polymer growth. The polypyrrole-coated iron electrodes were electroactive and remained highly conducting and stable in the ionic liquid. Using electrochemical impedance spectroscopy, the charge-transfer resistance of the uncoated iron electrode was determined as $1.25 \times 10^{4} \Omega \mathrm{cm}^{2}$ compared to the much lower resistance of $1.90 \times 10^{2} \Omega \mathrm{cm}^{2}$ measured for the highly conducting polypyrrolecoated electrode in the ionic liquid.

(C) 2004 The Electrochemical Society. [DOI: 10.1149/1.1831211] All rights reserved.
\end{abstract}

Manuscript submitted March 16, 2004; revised manuscript received June 11, 2004. Available electronically December 1, 2004.

The room temperature ionic liquid systems based on the $N, N^{\prime}$-dialkyl-substituted imidazolium cations have attracted increasing attention and interest in recent years. ${ }^{1-4}$ This is not surprising in light of their promising physical and chemical properties, which include high ionic conductivity, negligible volatility, nonflammability, excellent thermal and chemical stability, air and moisture stability, and, in terms of electrochemistry, a wide potential window.

Research into the formation, or properties, of conducting polymers in ionic liquids dates back to the mid 1980s, with the work of Osteryoung and co-workers. ${ }^{5-7}$ However, the chloroaluminate ionic liquids used in these investigations were moisture sensitive. In more recent times, it has been shown that conducting polymers can be electrosynthesized in the ambient temperature imidazolium-based ionic liquids with anions such as hexafluorophosphate or tetrafluoroborate. ${ }^{8-14}$ But of more significance is the fact that the conducting polymers cycled in, or electropolymerized from, these imidazolium-based ionic liquids exhibit greatly enhanced electrochemical stability, negligible polymer degradation and fast switching speeds. ${ }^{8-14}$ For example, Lu et al. ${ }^{10}$ found that polyaniline, polypyrrole, and polythiophene showed negligible loss in electroactivity when cycled in room-temperature ionic liquids composed of 1-butyl-3-methyl imidazolium cations with either tetrafluoroborate or hexafluorophosphate anions. Sekiguchi et al. ${ }^{13,14}$ used 1-ethyl-3methylimidazolium trifluoromethanesulfonate as an electrolyte for the electrochemical polymerization of pyrrole. The electrochemical capacity and conductivity of the polypyrrole were significantly increased when prepared from the ionic liquid. Other polymer systems that have been studied in these imidazolium-based ionic liquids include poly(3,4-ethylenedioxythiophene $)^{15,16}$ and poly(2-methoxy-5-(2'-ethylhexyloxy)-1,4-phenylene vinylene (MEH-PPV). ${ }^{17}$

These studies have all been conducted with inert electrodes. Although conducting polymers have been deposited at corrosion susceptible electrodes, from aqueous solutions, ${ }^{18,19}$ there are no reports on the preparation of conducting polymers at these substrates using ionic liquids as the solvent system. In this paper, results are presented and discussed on the electropolymerization of pyrrole from an ionic liquid at iron to generate adherent and stable conducting polypyrrole films.

\section{Experimental}

Synthesis and characterization of 1-butyl-3-methylimidazolium hexafluorophosphate $\left(\mathrm{bmimPF}_{6}\right)$.- -All chemical reagents were purchased from Aldrich. Bromobutane and hexafluorophosphoric acid were used without further purification. 1-methyl-imidazole and

\footnotetext{
* Electrochemical Society Active Member.

${ }^{\mathrm{z}}$ E-mail: cbbreslin@may.ie
}

pyrrole were distilled under vacuum prior to use. 1-butyl-3methylimidazolium hexafluorophosphate was synthesized according to the methods described elsewhere ${ }^{20}$ with some modifications. The precursor, 1-butyl-3-methylimidazolium bromide (bmimBr), was prepared by reacting equimolar amounts of bromobutane and 1-methyl-imidazole in toluene, for $3 \mathrm{~h}$, in a $500 \mathrm{~mL}$ round-bottomed flask fitted with a reflux condenser. The resulting viscous liquid was allowed to cool and washed five times with $100 \mathrm{~mL}$ aliquots of ethyl acetate. After the last washing any remaining ethyl acetate was removed under vacuum at $70^{\circ} \mathrm{C}$. To prepare bmimPF $\mathrm{F}_{6}$, hexafluorophosphoric acid was slowly added to bmimBr dissolved in distilled water over an ice bath in a 1.3:1 ratio. After stirring for $10 \mathrm{~h}$, water was decanted from the mixture and the remaining viscous ionic liquid was dissolved in dichloromethane which was then washed approximately ten times with water until neutral $\mathrm{pH}$ was achieved. Then, the mixture was cleaned and purified over activated alumina, the dichloromethane was removed and bmimPF$_{6}$ was dried under vacuum at $60^{\circ} \mathrm{C}$.

The ionic liquid was characterized using nuclear magnetic resonance (NMR) spectroscopy performed in DMSO. ${ }^{1} \mathrm{H}: 0.91[\mathrm{t}(3 \mathrm{H})]$, $1.28[\mathrm{~m}(2 \mathrm{H})], 1.78[\mathrm{~m}(2 \mathrm{H})], 3.88[\mathrm{~s}(3 \mathrm{H})], 4.23[\mathrm{t}(2 \mathrm{H})], 7.8[\mathrm{~d}(2 \mathrm{H})]$, 9.3[s (1H)]. ${ }^{31} \mathrm{P}:[\mathrm{sp}(6 \mathrm{~F})]$.

In order to rule out any possibilities of impurities in the melt, a second synthesis procedure was employed. This followed the procedure outlined by Wilkes et al. ${ }^{21}$ The bmimBr was prepared by refluxing 1-methyl imidazole with a large excess (1:3) of bromobutane for $24 \mathrm{~h}$. The excess bromoalkane was removed, and the crude product was recrystallized from ethyl acteate over $48 \mathrm{~h}$, which was then evaporated under reduced pressure. The bmimPF $_{6}$ was then prepared by adding hexafluorophosphoric acid and purified as above. The viscous liquid was dried under vacuum at $60^{\circ} \mathrm{C}$. In the remaining text this method of preparation is referred to as the second method of synthesis.

In the case of both synthetic methods, the water content was measured using Karl-Fischer coulometry (Metrohm 831 coulometer). The water content varied between 10 and $100 \mathrm{ppm}$ depending on the extent of drying. For the electrochemistry experiments carried out under an atmosphere of nitrogen, the ionic liquid was dried and degassed on a Schlenk line and then the electrochemistry was performed under an atmosphere of dried nitrogen in a glove bag. The water content in these experiments was approximately $10 \mathrm{ppm}$.

Electropolymerization of pyrrole and characterization of polymer.-Electrodes were prepared from pure iron (99.995\%) and pure platinum (99.99\%). The electrodes were provided in rod form $(0.3 \mathrm{~cm}$ in diameter). These rods were embedded in epoxy resin in a Teflon holder with electrical contact being achieved by means of a copper wire threaded into the base of the metal sample. Prior to each test, the exposed surfaces were polished to a smooth surface finish, 


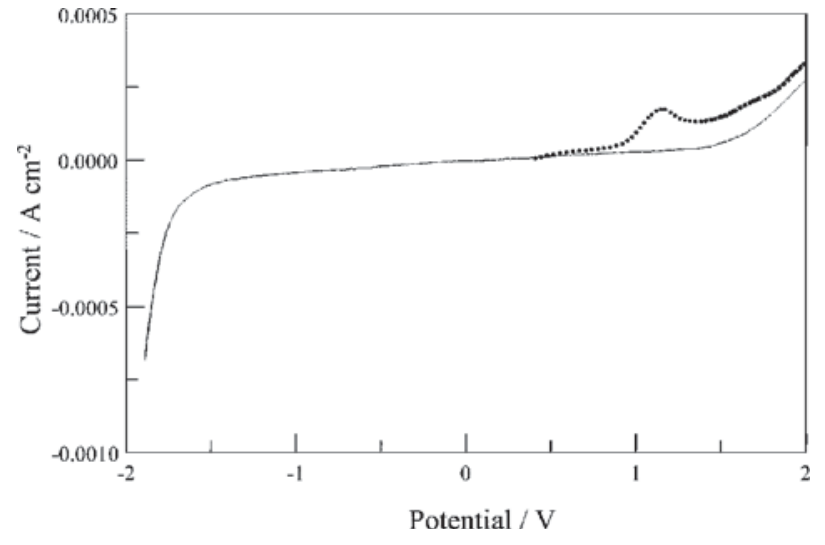

Figure 1. Linear sweep voltammogram recorded at $50 \mathrm{mV} \mathrm{s}^{-1}$ for platinum polarized in (-) neat bmimPF$_{6}$ and (---) bmimPF $_{6}$ saturated with bromide.

using 1200 grit $\mathrm{SiC}$ and rinsed with distilled water. Platinum wire was used as the auxiliary electrode and a silver wire was used as a quasi-reference electrode. All potentials quoted are relative to this electrode. All electropolymerization reactions were carried out using $5 \mathrm{~mL}$ of neat bmimPF $\mathrm{F}_{6}$. Unless otherwise stated, the iron electrode was first polarized at $1.3 \mathrm{~V}$ for $5 \mathrm{~min}$ in neat $\operatorname{bmimPF}_{6}$, then the electropolymerization reaction was performed at a constant potential of $1.3 \mathrm{~V}$ in $0.3 \mathrm{~mol} \mathrm{dm}^{-3}$ pyrrole dissolved in bmimPF $_{6}$.

Electrochemical measurements were carried out using an EG\&G potentiostat, model 263, a Solartron EI 1287 electrochemical interface and a Solartron 1250 frequency response analyser. Electrochemical impedance measurements were recorded at open-circuit potential with an excitation voltage of $10 \mathrm{mV}$. All impedance data were fit to appropriate equivalent circuits using a complex nonlinear least square fitting routine, using both the real and imaginary components of the data.

Light microscopy was performed on an Olympus light microscope, model BX51M, coupled with a ColorView soft imaging system. Iron electrodes used for light microscopy were prepared from iron supplied in sheet form. Spectroelectrochemistry measurements were recorded on a Varian Cary $50 \mathrm{UV}$-spectrometer coupled to a Solartron EI 1287 electrochemical interface. In these measurements the working electrode was an indium tin oxide (ITO) electrode (resistivity of $20 \Omega$ ), immersed in neat bmimPF $F_{6}$. A quartz UV-cell, 1 $\mathrm{cm}$ in path length, was used. Fourier transform infrared (FTIR) spectra were recorded using a Shimadzu AIM-8800 Infrared Microscope.

\section{Results and Discussion}

Growth of polypyrrole.-Prior to any electropolymerization studies, the electrochemical window of the ionic liquid was obtained. For the purpose of these experiments, pure platinum was cycled in neat bmimPF 6 at $50 \mathrm{mV} \mathrm{s}^{-1}$. The electrochemical window was found to extend from about -1.75 to $+1.75 \mathrm{~V}$ showing that the ionic liquid is pure and not prone to any decomposition reactions within this potential interval. Typical data are shown in Fig. 1 for the ionic liquid synthesized according to the first method described in the Experimental section. Similar data were recorded for the ionic liquid synthesized using the second method showing little difference between both methods. Also, shown on this plot is the melt saturated with bromide. Oxidation of bromide to bromine is seen clearly at $1.15 \mathrm{~V}$ for the trace recorded in the ionic liquid saturated with bromide. It should be noted that the solubility of $\mathrm{NaBr}$ in the ionic liquid is very low showing that the ionic liquid is relatively free from any unreacted bromide.

Polypyrrole coatings were deposited at iron electrodes from neat bmimPF $_{6}$. The iron electrodes were first pretreated by polarization at $1.3 \mathrm{~V}$ for $5 \mathrm{~min}$ in neat $\mathrm{bmimPF}_{6}$. This procedure was employed

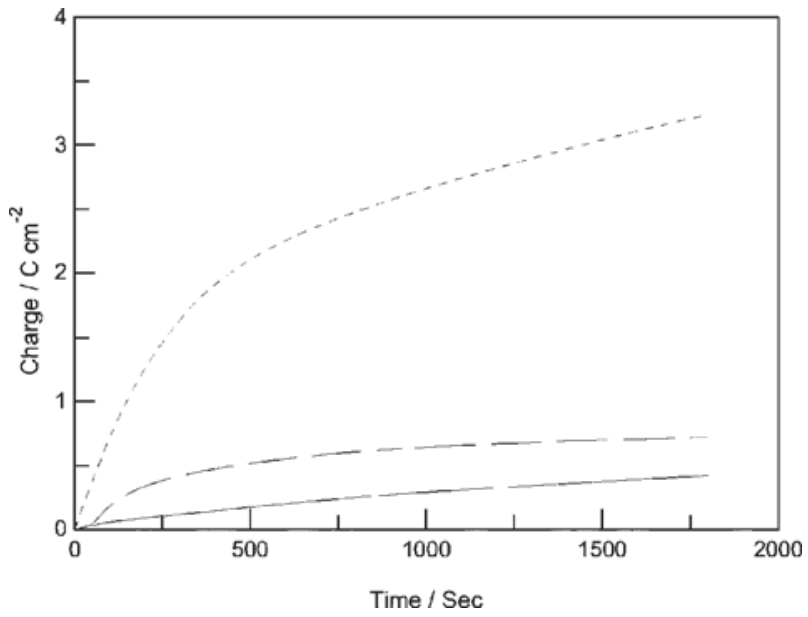

Figure 2. Cumulative charge passed for polarization of iron at $1.3 \mathrm{~V}$ in bmimPF $_{6}(100 \mathrm{ppm}$ water $)$ in (- $-(-)$ the absence of pyrrole; (-) $0.1 \mathrm{~mol} \mathrm{dm}^{-3}$ pyrrole, and (- - -) $0.2 \mathrm{~mol} \mathrm{dm}^{-3}$ pyrrole.

to minimize the dissolution of iron during the subsequent electropolymerization reaction. Pyrrole monomer was then added to the ionic liquid and the electrode was polarized for a further $30 \mathrm{~min}$ in the monomer-containing bmimPF $\mathrm{P}_{6}$. The effects of monomer concentration and electropolymerization potential on the growth of polypyrrole at iron are evident from the data presented in Fig. 2 and 3. In Fig. 2, the cumulative charge passed as a function of time for a total polarization time of $30 \mathrm{~min}$ is shown. Three traces, typical of the growth profiles recorded in the presence of 0.1 and $0.2 \mathrm{~mol} \mathrm{dm}{ }^{-3}$ pyrrole and the charge associated with the dissolution of iron in the absence of pyrrole, are shown. In Fig. 3, the total charge passed following a 30 min electropolymerization period in $0.3 \mathrm{~mol} \mathrm{dm}^{-3}$ pyrrole is shown as a function of the electropolymerization potential.

As expected, the charge passed and amount of polymer deposited increase with increasing monomer concentration and increasing applied potential. At a pyrrole concentration of $0.1 \mathrm{~mol} \mathrm{dm} \mathrm{dm}^{-3}$ pyrrole, the total charged passed following $30 \mathrm{~min}$ was $0.4 \mathrm{C} \mathrm{cm}^{-2}$, Fig. 2 . But, increasing the monomer concentration to $0.2 \mathrm{~mol} \mathrm{dm}^{-3}$ gave rise to a significant charge increase to $3.2 \mathrm{C} \mathrm{cm}^{-2}$, Fig. 2. Although not shown on the plot, a further increase in the concentration of pyrrole has very little influence on the rate of polymer growth, with the charge passed following $30 \mathrm{~min}$ being typically $3.1 \pm 0.2 \mathrm{C}$ $\mathrm{cm}^{-2}$ for pyrrole concentrations between 0.2 and $0.4 \mathrm{~mol} \mathrm{dm}{ }^{-3}$. These results indicate that above a critical concentration of $0.2 \mathrm{~mol}$ $\mathrm{dm}^{-3}$ pyrrole, the concentration of the monomer has little influence on the rate of electropolymerization in the ionic liquid. In addition, it can be concluded from Fig. 3, that a significant increase in the rate

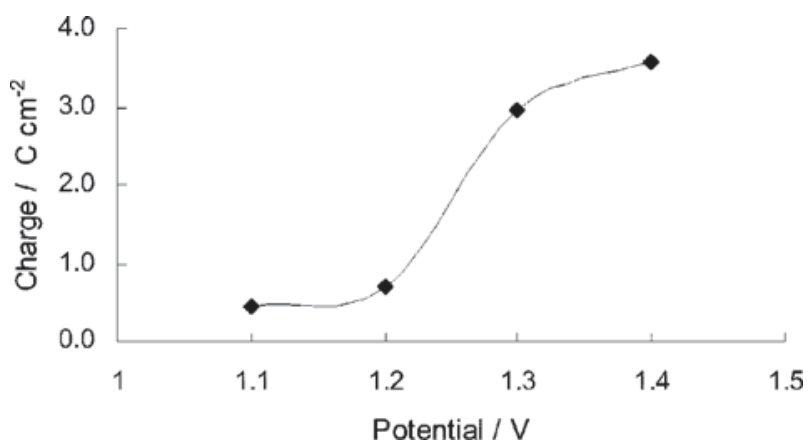

Figure 3. Total charge passed over a 30 min polarization period plotted as a function of applied potential for electropolymerization of pyrrole at iron in a $0.3 \mathrm{~mol} \mathrm{dm}{ }^{-3}$ pyrrole solution in bmimPF $_{6}$ (100 ppm water). 
of polymer growth occurs on increasing the potential from 1.2 and $1.3 \mathrm{~V}$. Also, it is interesting to note that dissolution of the iron

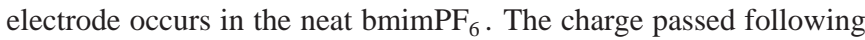
30 min of polarization, in the absence of the monomer, is approximately $0.70 \mathrm{C} \mathrm{cm}^{-2}$. This is higher than that observed with the lower $0.1 \mathrm{~mol} \mathrm{dm}^{-3}$ pyrrole-containing solution. This indicates that the polypyrrole layer, once nucleated, limits the dissolution of the iron substrate. Using the assumption that $240 \mathrm{mC} \mathrm{cm}{ }^{-2}$ produces a $1.0 \mu \mathrm{m}$ thick polypyrrole film, ${ }^{11}$ the thickness of the polypyrrole films produced at $1.3 \mathrm{~V}$ for $30 \mathrm{~min}$ can be approximated as 9.0 $\pm 1.0 \mu \mathrm{m}$. In this analysis, the charge due to the oxidation of the iron substrate was approximated as $0.5 \mathrm{C} \mathrm{cm}^{-2}$. Similar results were obtained regardless of the method used in synthesizing the ionic liquid. However, the rate of electropolymerization was dependent on the concentration of water in the ionic liquid, with lower growth rates being observed in the very dry ionic liquids with water contents at or below $10 \mathrm{ppm}$.

These findings agree well with results presented in the literature on the growth of polypyrrole from aqueous media at corrosion susceptible electrodes. ${ }^{22-24}$ For example, the rate of electropolymerization of pyrrole was found to increase with increasing monomer concentration for deposition of polypyrrole at copper ${ }^{22}$ and steel, ${ }^{23}$ while Asavapiriyanont et al. ${ }^{24}$ have reported an increase in the polymer deposition current with increasing potential. However, the induction period, which is normally observed during the deposition of conducting polymers at active metals in aqueous media ${ }^{22,23}$ appears to be absent, or at least is very short, in the case of polymer deposition from bmimPF $\mathrm{F}_{6}$, Fig. 2. In aqueous solution, this induction time is usually attributed to the formation of an iron salt with the acid used in the electropolymerization reaction, which is a necessary first step before deposition of the polymer occurs. ${ }^{22,23}$ Although dissolution of iron occurs at $1.3 \mathrm{~V}$ in the ionic liquid, there was little evidence from the electrochemical data to indicate the formation of a salt layer at the iron electrode prior to the deposition of the polymer. Also, the higher potentials used in this work, compared to some of the reports in aqueous solution, reflect the increased IR drop in ionic liquids due to their higher viscosity. ${ }^{25}$

Characterization and morphology of polypyrrole.-The growth of polypyrrole from bmimPF $\mathrm{P}_{6}$ was monitored using a spectroelectrochemical technique. In these experiments, the electropolymerization potential and monomer concentration were reduced to $1.0 \mathrm{~V}$ and $0.1 \mathrm{~mol} \mathrm{dm}{ }^{-3}$ pyrrole, respectively, in order to decrease the rate of polymer deposition at the ITO electrode. Shown in Fig. 4a are the UV-vis spectra recorded following 5 and 10 min periods of electropolymerization. An increase in the absorbance across the entire visible region is seen on increasing the electropolymerization period from 5 to $10 \mathrm{~min}$. This corresponds to the presence of a thicker polymer layer at the ITO electrode and coincides with an increase in the charge passed in the electrochemical data recorded. This spectroelectrochemical response, across the entire visible region, has been reported previously by Fermín et al. ${ }^{26}$ However, with continued polymer growth a broad peak becomes evident at low wavelengths ( $\lambda$ max at approximately $450 \mathrm{~nm}$ ) and at wavelengths higher than $600 \mathrm{~nm}$. This gradual absorbance increase above $600 \mathrm{~nm}$ has been attributed to the existence of the bipolaron state of the polymer. $^{27,28}$ This is consistent with the polymer being oxidized at $1.0 \mathrm{~V}$. Fermín et al. ${ }^{26}$ have reported an absorbance band near 425 $\mathrm{nm}$ and have attributed this to the neutral form of polypyrrole. However, it is unlikely that the absorbance maximum seen at $450 \mathrm{~nm}$ in this work is entirely due to the neutral species of polypyrrole, given the high potentials used for electropolymerization. Instead, the absorption band is more likely due to electron transition from the valence to the antipolaron band, as reported by Bredas et al. ${ }^{28}$

Shown in Fig. 4b is a plot of the natural logarithm of the absorbance at $450 \mathrm{~nm}$ as a function of time. From these data it can be seen that the electropolymerization of pyrrole from bmimPF $_{6}$ follows a two-stage process, with each stage obeying first-order kinetics. The rate constants, calculated from the slopes of the plots, were
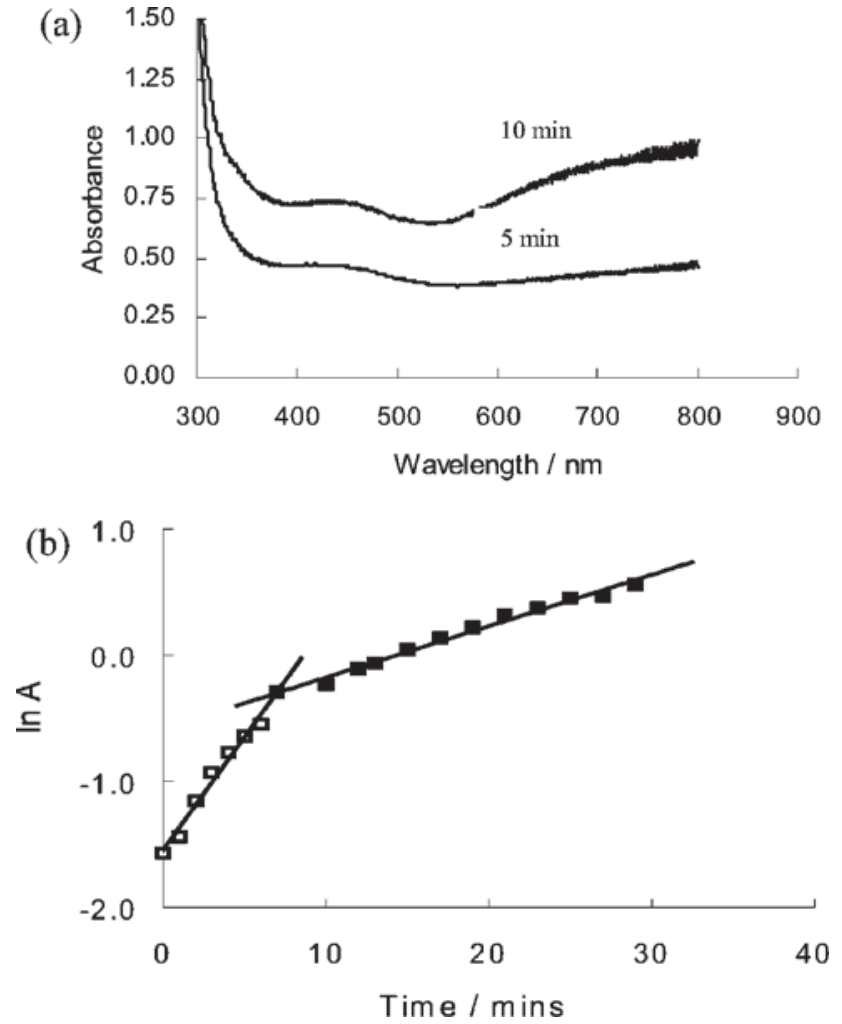

Figure 4. (a) UV-vis spectra recorded following 5 and 10 min periods during the electropolymerization of pyrrole at an ITO electrode at $1.0 \mathrm{~V}$ in 0.1 mol dm ${ }^{-3}$ pyrrole in bmimPF b $_{6}(100$ ppm water). (b) First-order kinetics plots showing two phases of polypyrrole growth: $(\square \square \square)$ phase 1 and phase 2 .

determined as $0.18 \mathrm{~min}^{-1}$ for stage I, which corresponds to about the first $6 \mathrm{~min}$ of reaction and $0.04 \mathrm{~min}^{-1}$ for stage II, which extends between approximately 8 and $30 \mathrm{~min}$. The rate of the reaction is higher for stage I, which corresponds to the nucleation and initial growth of the polymer. The rate of the electropolymerization reaction then appears to slow down to a steady growth phase, which dominates the remainder of the electropolymerization process.

An IR spectrum recorded for a polypyrrole-coated iron electrode is presented in Fig. 5a. The polypyrrole layer was deposited at $1.3 \mathrm{~V}$ from neat bmimPF ${ }_{6}$ containing $0.3 \mathrm{~mol} \mathrm{dm}^{-3}$ pyrrole. The observed bands are characteristic of polypyrrole and were assigned according to literature reports. ${ }^{29-32}$ The assignment of the bands reveals the $\mathrm{N}-\mathrm{H}$ in-plane ring deformation at $1046 \mathrm{~cm}^{-1}$, C-H out of plane deformations at 920 and $739 \mathrm{~cm}^{-1}$ and the C-C out of plane deformations at $966 \mathrm{~cm}^{-1}$. The pyrrole ring stretches can be seen at 1165 , 1463 , and $1540 \mathrm{~cm}^{-1}$. The intense band at $815 \mathrm{~cm}^{-1}$ was assigned to the $\mathrm{PF}_{6}^{-}$anions of the ionic liquid. This was deduced by recording the IR spectrum of the neat ionic liquid and $\mathrm{NaPF}_{6}$, which are shown in Fig. 5b and c, respectively. The IR spectrum of $\mathrm{NaPF}_{6}$ gives an intense peak at $828 \mathrm{~cm}^{-1}$, which is also evident in the ionic liquid. This is consistent with doping of the polypyrrole film by the ionic liquid, as these films were well rinsed with acetone and any remaining acetone was allowed to evaporate prior to analysis, thus minimizing any traces of free ionic liquid.

A high-resolution optical micrograph of a polypyrrole-coated iron electrode is shown in Fig. 6. Again, the polymer was deposited at $1.3 \mathrm{~V}$ from neat bmimPF 6 containing $0.3 \mathrm{~mol} \mathrm{dm}^{-3}$ pyrrole and the surface was rinsed with acetone before analysis. The data shown in Fig. 6 are characteristic of a homogenous polypyrrole layer, with microspheroidal aggregates of polypyrrole. Although it is not clearly obvious from this micrograph, these polypyrrole layers are smoother than those grown from aqueous systems. ${ }^{22}$ It has been documented 


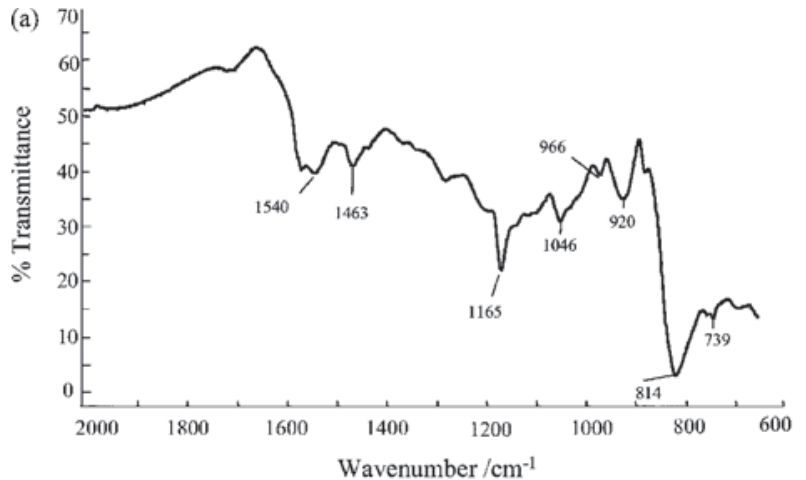

(b)
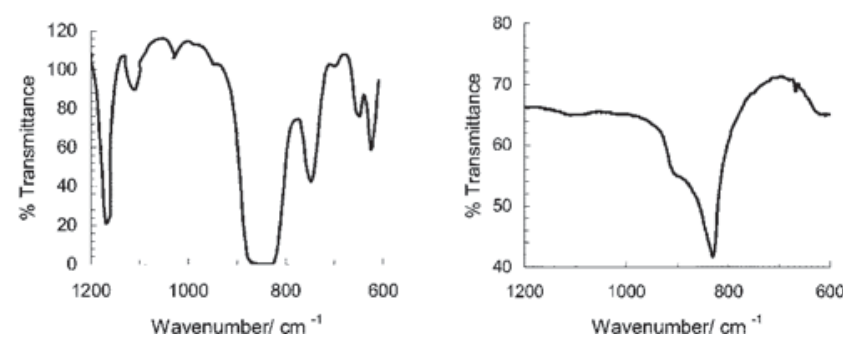

Figure 5. FTIR spectrum recorded for (a) polypyrrole-coated iron, (b) bmimPF 6 , and (c) $\mathrm{NaPF}_{6}$.

previously that polypyrrole layers grown at platinum from ionic liquids appear smoother than those grown from conventional aqueous systems. ${ }^{13,25}$ For example, Sekiguchi et al. ${ }^{13}$ studied the morphology of polypyrrole grown from $\mathrm{CH}_{3} \mathrm{CN}$ and $\mathrm{EMICF}_{3} \mathrm{SO}_{3}$ and found that the more conductive ionic liquid gave rise to smoother films, while Pringle et al. ${ }^{25}$ report that the more viscous ionic liquids produce the smoothest polypyrrole layers.

Electrochemical behavior of polypyrrole.-The electroactivity of the polypyrrole-coated iron electrode was investigated in deoxygenated bmimPF ${ }_{6}$ using cyclic voltammetry. In addition, the ionic liquid was dried to water contents at or below $10 \mathrm{ppm}$. For comparison purposes, the electrochemical behavior of polypyrrole-coated plati-

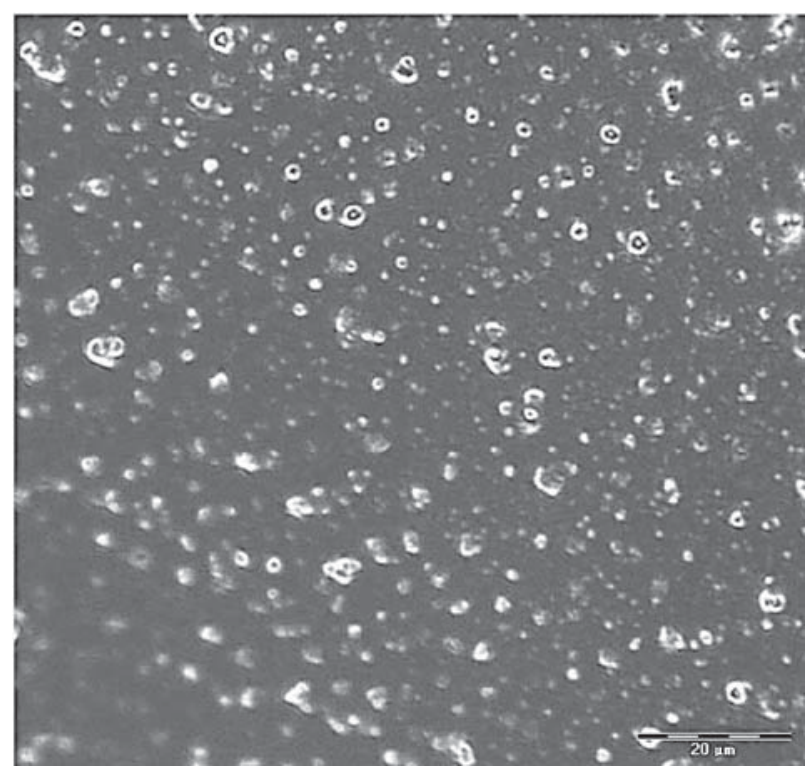

Figure 6. Optical micrograph of polypyrrole-coated iron.
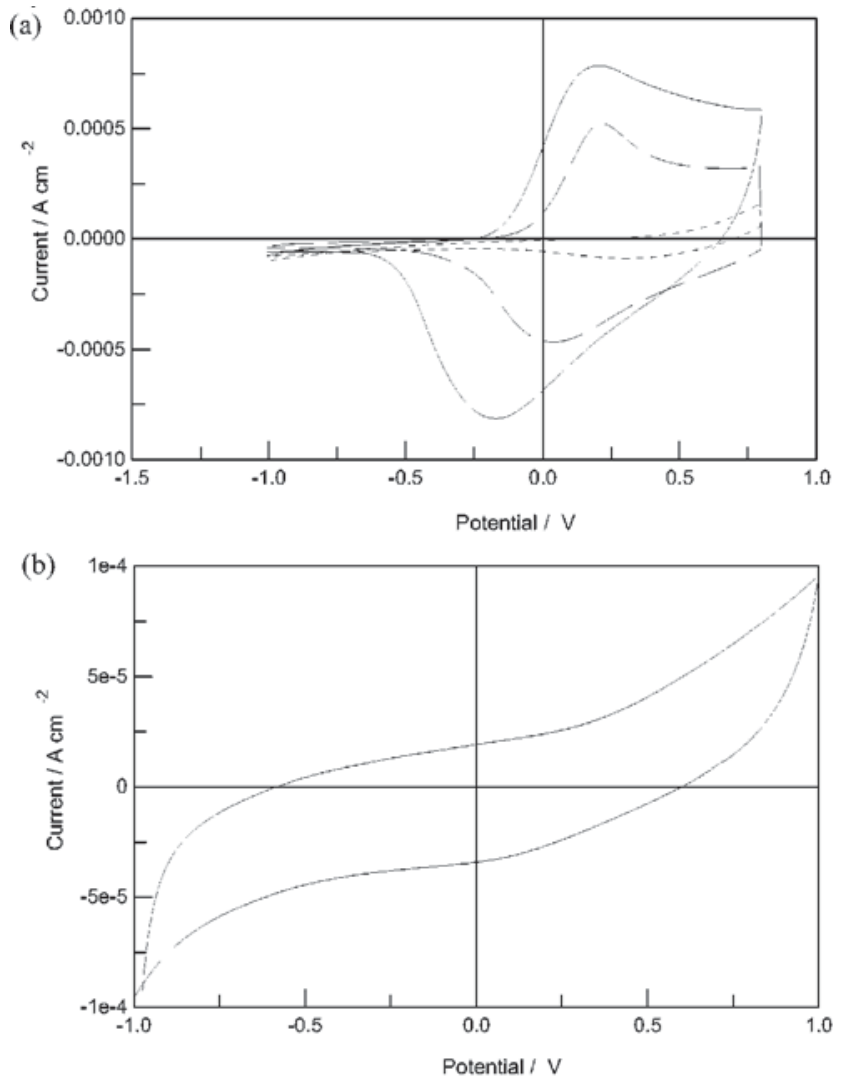

(c)

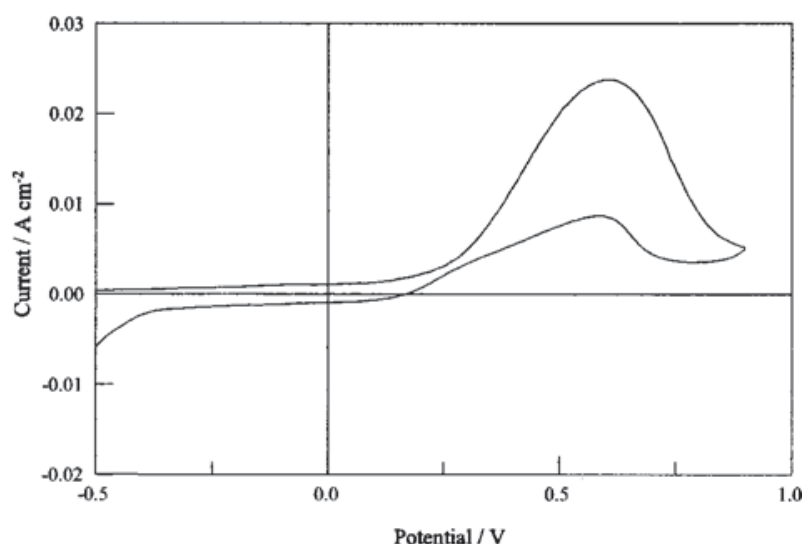

Figure 7. Cyclic voltammograms recorded at $50 \mathrm{mV} \mathrm{s}^{-1}$ in $\operatorname{bmimPF}_{6}(10$ ppm water) for (a) ( $-\longrightarrow$ ) polypyrrole-coated platinum prepared at $1.0 \mathrm{~V},(---)$ polypyrrole-coated platinum prepared at $1.3 \mathrm{~V}$, and $(-)$ polypyrrole-coated iron, (b) pure uncoated platinum, and (c) uncoated iron.

num was also investigated. The polymers were deposited at $1.3 \mathrm{~V}$ with $0.3 \mathrm{~mol} \mathrm{dm}^{-3}$ pyrrole dissolved in bmimPF $_{6}$ for the iron system and at 1.3 and $1.0 \mathrm{~V}$ for the platinum system. The cyclic voltammograms were recorded at $50 \mathrm{mV} \mathrm{s}^{-1}$ between the potential limits of -1.0 and $0.8 \mathrm{~V}$ in a dry nitrogen environment. These cyclic voltammograms recorded for polypyrrole-coated iron and the polypyrrole-coated platinum electrodes are shown in Fig. 7a, while similar data recorded for uncoated platinum and uncoated iron cycled in deoxygenated bmimPF $_{6}$ are presented in Fig. $7 \mathrm{~b}$ and $c$ respectively.

It is interesting to note that the polypyrrole-coated platinum electrode deposited at $1.3 \mathrm{~V}$ does not exhibit electroactive behavior, Fig. 7a. This is consistent with overoxidation of the polymer during growth at the relatively high potential of $1.3 \mathrm{~V}$. However, when the polymer is formed at the lower potential of $1.0 \mathrm{~V}$, clear oxidation/ 


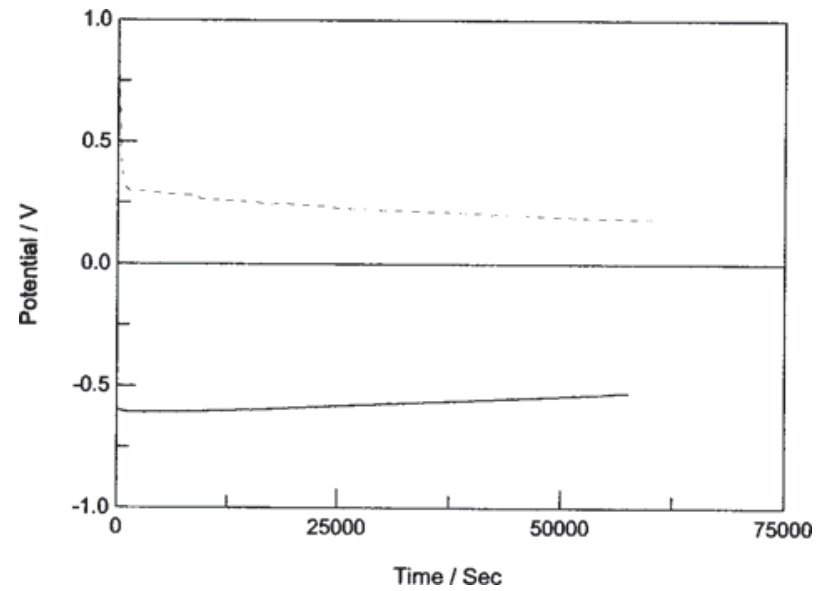

Figure 8. Open-circuit potential recorded as a function of time for ( - ) uncoated iron and (---) polypyrrole-coated iron immersed in bmimPF (100 ppm water).

reduction waves are seen indicating oxidation and reduction of the polypyrrole. An examination of the trace recorded for polypyrrolecoated iron shows an oxidation peak at $0.18 \mathrm{~V}$. This corresponds to the oxidation of polypyrrole and is confirmed by the presence of a clear oxidation wave at similar potentials for the electroactive polypyrrole-coated platinum. On the reverse sweep, a broad cathodic wave with a peak potential near $-0.2 \mathrm{~V}$ is observed. This can be attributed to the reduction of the polypyrrole film. Although, the polypyrrole was electrosynthesized at $1.3 \mathrm{~V}$ at the iron substrate, the polymer is electroactive and conducting. This indicates that the polypyrrole is not susceptible to any significant amount of overoxidation when electropolymerized at the iron substrate at $1.3 \mathrm{~V}$. No redox activity is observed on cycling pure platinum in the dry deoxygenated ionic liquid, Fig. 7b, but oxidation of iron is seen, at potentials higher than about $0.3 \mathrm{~V}$, Fig. 7c. However, when the polypyrrole-coated iron electrode is cycled in the ionic liquid, there is no evidence of iron dissolution, suggesting that the polypyrrole layer protects the iron substrate from corrosion. On comparing the traces recorded for the polypyrrole-coated platinum and polypyrrole-coated iron electrodes, it is evident that the polymer formed at iron appears to exhibit the greater electroactivity. However, this is connected with the thickness of the polymers, with a thicker polymer being formed at the iron substrate.

This polypyrrole redox couple has been documented previously in ionic liquids. ${ }^{9,13,25}$ Mazurkiewicz et al. ${ }^{9}$ have shown the presence of the polypyrrole redox couple in bmimPF $_{6}$ with the oxidation wave centered near $0.2 \mathrm{~V}$ and the reduction wave centered at -0.3 V. These values agree well with the peak potentials in Fig. 7a. Also, it has been shown that conducting polymers cycled in ionic liquids, such as bmimPF$_{6}$, exhibit increased stability during redox cycling. ${ }^{8-14}$ For example, Mazurkiewicz et al. ${ }^{9}$ have shown that polypyrrole is considerably more stable when cycled in bmimPF $_{6}$ than in an aqueous tetrabutylammonium hexafluorophosphate electrolyte. A similar effect was observed in these experiments; the peak potentials and peak currents remained relatively constant with cycling.

In order to probe further the stability of the polypyrrole-coated iron electrode, the open-circuit potential and the impedance response of the polypyrrole-coated iron and uncoated iron electrodes were monitored as a function of time in $\mathrm{bmimPF}_{6}$. Typical data from these measurements are shown in Fig. 8, 9, and 10. In Fig. 8, the open-circuit potential values recorded over a period of $16 \mathrm{~h}$ for both systems are shown. The uncoated iron electrode adopts a steady-state potential near $-560 \mathrm{mV}$. However, the polypyrrolecoated iron electrode adopts a potential, some $750 \mathrm{mV}$ more noble, with a steady-state value near $200 \mathrm{mV}$. The initial sharp drop in the potential from about 750 to $200 \mathrm{mV}$ is probably connected with
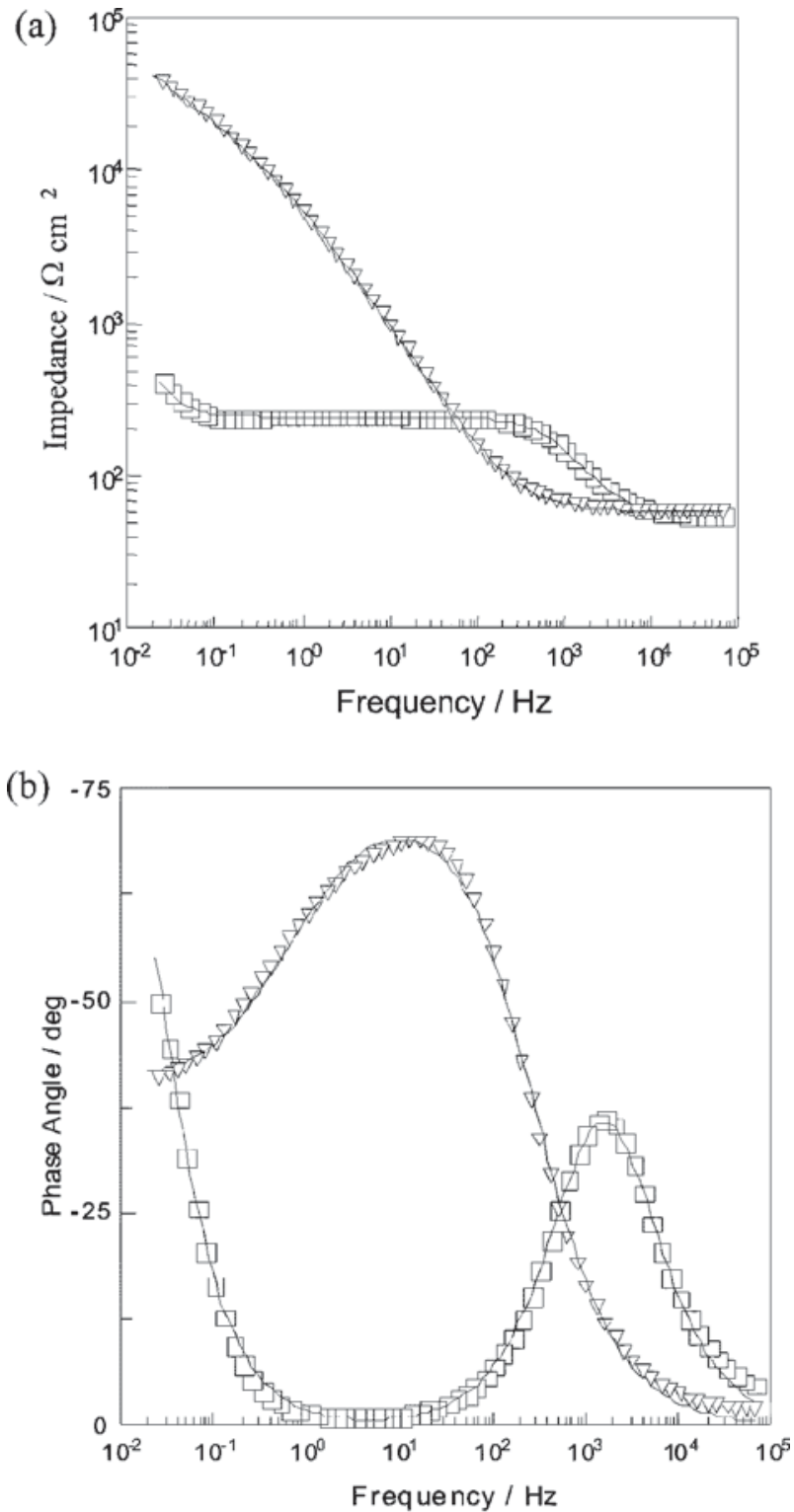

(c)

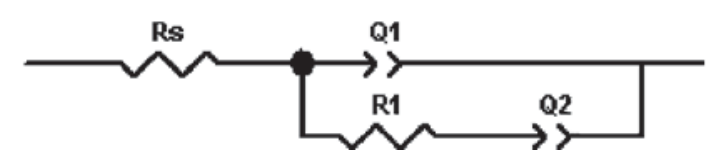

Figure 9. (a) and (b) Impedance spectra recorded for ( $\square \square \square \square$ ) polypyrrolecoated iron and $(\triangle \triangle \triangle \triangle)$ uncoated iron, following 490 min under opencircuit conditions in bmimPF $\mathrm{P}_{6}$ (100 ppm water). (c) Equivalent circuit used to model impedance data.

water in the ionic liquid and the oxidation of the iron substrate. These results indicate that not only are these electrodes stable for periods greater than $16 \mathrm{~h}$ in bmimPF $_{6}$, but also the obvious ennoblement in the potential indicates that the coating protects the metal from dissolution, shifting the open-circuit potential into the passive region for iron.

The clear stability of the polypyrrole-coated iron electrode in bmimPF$_{6}$ was also evident from the impedance response. Typical Bode plots for the polypyrrole-coated iron and the uncoated iron electrodes are shown in Fig. 9. These data were recorded under open-circuit conditions following $490 \mathrm{~min}$ immersion in the ionic liquid. The symbols represent the experimental data while the con- 


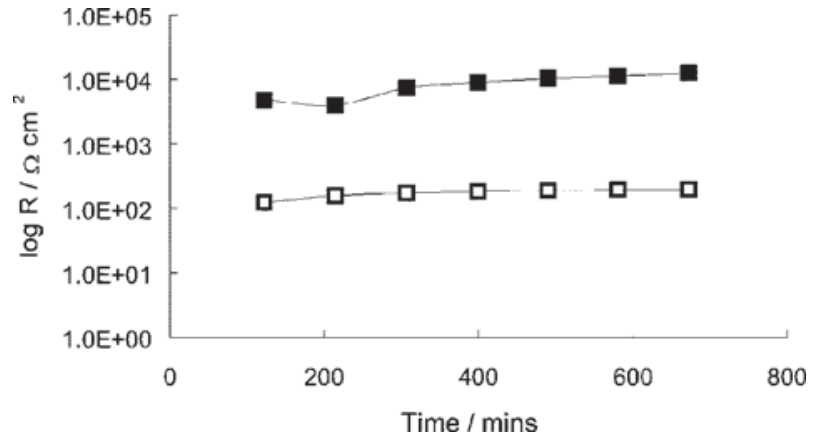

Figure 10. Plot of logarithm of $\mathrm{R}$ as a function of time for ( $\square \square \square \square$ ) polypyrrole-coated iron and $(\mathbf{\square} \mathbf{\square})$ uncoated iron in bmimPF $\mathrm{b}_{6}(100 \mathrm{ppm}$ water).

tinuous trace represents the simulated data obtained using the equivalent circuit shown in Fig. 9c. In these equivalent circuits, $R$ refers to resistance elements while $Q$ indicates a constant phase element. The impedance of a constant-phase element is defined as $Z_{\mathrm{CPE}}=\left[Q(j \omega)^{n}\right]^{-1}$ where $-1 \leqslant n \leqslant 1 .{ }^{33}$ In the special case where $n$ is equal to $1.0, Q$ is equivalent to an ideal capacitor with a phase angle of $90^{\circ}$. Although, the impedance responses of the polypyrrole-coated and uncoated iron electrodes are considerably different, the data could be fit to the same equivalent circuit, Fig. 9c. In this analysis, the resistor, $R s$, represents the solution resistance of the ionic liquid and adopts values in the region of $55 \pm 5 \Omega \mathrm{cm}^{2}$. This is then followed in series by a constant phase element, $Q 1$, and a second resistor $R_{1}$. In this parallel couple, $Q 1$ represents the double-layer capacitance, which exists at the metal-ionic liquid interface, and $R 1$ is the charge-transfer resistance. Typical values of these parameters for the uncoated iron electrode are $30 \mu \mathrm{F} \mathrm{cm}^{-2}$ and $1.25 \times 10^{4} \Omega \mathrm{cm}^{2}$. This high charge-transfer resistance indicates stability of the uncoated iron electrode under open-circuit conditions in the ionic liquid. In the case of the polypyrrole-coated iron electrode, $Q 1$ adopts values of $30 \mu \mathrm{F} \mathrm{cm}{ }^{-2}$, typical of a double-layer capacitance and similar to the values calculated for the uncoated iron system. However, the charge-transfer resistance is considerably different attaining values of $190 \Omega \mathrm{cm}^{2}$, a significant reduction from the value of $1.25 \times 10^{4} \Omega \mathrm{cm}^{2}$ recorded for the uncoated system. This considerable reduction in the charge-transfer resistance is consistent with a highly conducting polypyrrole coating. ${ }^{18,19}$ The second constant phase element included in the circuit, $Q 2$, has an $n$ value of 0.46 for the uncoated iron electrode, indicating a diffusion process, and a value of unity for the polypyrrole-coated iron electrode signifying a capacitive term with a high capacitance of $19 \mathrm{mF}$ $\mathrm{cm}^{-2}$. Again, this is consistent with a highly conducting polypyrrole coating. Indeed, such high capacitance values have been attributed previously to the intrinsic redox charging of conducting polymer films in aqueous media. ${ }^{18,19}$ These impedance data show clearly that the polypyrrole coating formed at the iron electrode is conducting, in agreement with the voltammetry data presented in Fig. 7.

It is interesting to note that these polypyrrole coatings deposited at iron remain stable and conducting on continued immersion in the ionic liquid. This is clearly evident from the data presented in Fig. 10 , which show the charge-transfer resistance plotted as a function of time for polypyrrole-coated and uncoated iron immersed, under open-circuit conditions, in bmimPF $_{6}$. Again, the considerable 100fold reduction in the charge-transfer resistance, $R$, of the polypyrrole-coated electrode compared to the uncoated iron electrode is evident. Interestingly, the polymer resistance remains low for the duration of the experiment indicating that the polypyrrolecoated iron electrode is stable and remains highly conducting. Furthermore, as shown by Grgur et al., ${ }^{34}$ dissolution of iron through the pores of a conducting polymer coating in aqueous system, results in a significant increase in the impedance of the system. As shown in Fig. 10, this is not the case here, as the resistance remains low for the duration of the experiment.

\section{Conclusions}

Adherent conducting polypyrrole films were deposited at pure iron from bmimPF $\mathrm{F}_{6}$ in the potential range of 1.0 to $1.4 \mathrm{~V}$ with pyrrole concentrations between 0.1 and $0.3 \mathrm{~mol} \mathrm{dm}^{-3}$. An increase in the pyrrole concentration and the applied potential gave rise to an increase in the rate of electropolymerization. Spectroelectrochemical measurements showed that the growth of the polypyrrole film followed a two-step process, with both steps obeying first-order kinetics. The deposited polypyrrole layers were electroactive and remained highly conducting during immersion in the ionic liquid.

\section{Acknowledgments}

The authors gratefully acknowledge the support of this work by Enterprise Ireland (IF/2002/0003).

National University of Ireland Maynooth assisted in meeting the publication costs of this article.

\section{References}

1. S. S. Palimkar, S. A. Siddiqui, T. Daniel, R. J. Lahoti, and K. V. Srinivasan, J. Org Chem., 68, 9371 (2003).

2. T. Itoh, N. Ouchi, Y. Nishimura, H. S. Hui, N. Katada, M. Niwa, and M. Onaka, Green Chem. 5, 494 (2003)

3. L. G. Lin, Y. Wang, J. W. Yan, Y. Z. Yuan, J. Xiang, and B. W. Mao, Electrochem. Commun., 5, 995 (2003).

4. J. H. Shin, W. A. Henderson, and S. Passerini, Electrochem. Commun., 5, 1016 (2003).

5. P. G. Pickup and R. A. Osteryoung, J. Electroanal. Chem., 195, 271 (1985).

6. T. A. Zawodzinskita, L. Janiszewska, and R. A. Osteryoung, J. Electroanal. Chem., 255, 111 (1988)

7. J. F. Oudard, R. D. Allendoerfer, and R. A. Osteryoung, J. Electroanal. Chem., 241, 231 (1988).

8. D. Z. Zhou, G. M. Spinks, G. G. Wallace, C. Tiyapiboonchaiya, D. R. MacFarlane, M. Forsyth, and J. Z. Sun, Electrochim. Acta, 48, 2355 (2003).

9. J. H. Mazurkiewicz, P. C. Innis, G. G. Wallace, D. R. MacFarlane, and M. Forsyth, Synth. Met., 135, 31 (2003)

10. W. Lu, A. G. Fadeev, B. H. Qi, E. Smela, B. R. Mattes, J. Ding, G. M. Spinks, J. Mazurkiewicz, D. Z. Zhou, G. G. Wallace, D. R. MacFarlane, S. A. Forsyth, and M. Forsyth, Science, 297, 983 (2002).

11. D. L. Boxall and R. A. Osteryoung, J. Electrochem. Soc., 151, E41 (2004).

12. W. Lu, A. G. Fadeev, B. Qi, and B. R. Mattes, J. Electrochem. Soc., 151, H33 (2004).

13. K. Sekiguchi, M. Atobe, and T. Fuchigami, Electrochem. Commun., 4, 881 (2002)

14. K. Sekiguchi, M. Atobe, and T. Fuchigami, J. Electroanal. Chem., 557, 1 (2003).

15. H. Randriamahazaka, C. Plesse, D. Teyssie, and C. Chevot, Electrochem. Commun., 5, 613 (2003).

16. H. Randriamahazaka, C. Plesse, D. Teyssie, and C. Chevot, Electrochem. Commun., 6, 299 (2004).

17. C. Yang, O. Sun, J. Qiao, and Y. Li, J. Phys. Chem. B, 107, 12981 (2003).

18. E. Musiani, Electrochim. Acta, 35, 1665 (1990).

19. E. S. Matveeva and M. J. Gonzalez-Tejera, J. Electrochem. Soc., 147, 1213 (2000).

20. J. G. Huddleston, H. D. Willauer, R. P. Swatloski, A. E. Visser, and R. D. Rodgers, Chem. Commun. (Cambridge), 16, 1765 (1998).

21. J. S. Wilkes, J. A. Levisky, R. A. Wilson, and C. L. Hussey, Inorg. Chem., 1982 1263

22. A. M. Fenelon and C. B. Breslin, Electrochim. Acta, 47, 4467 (2002).

23. W. Su and J. Iroh, J. Appl. Polym. Sci., 65, 417 (1997).

24. S. Asavapiriyanont, C. K. Chandler, G. A. Gunawardena, and D. Pletcher, J. Elec troanal. Chem., 177, 229 (1984).

25. J. Pringle, J. Efthimiadis, P. C. Howlett, J. Efthimiadis, D. R. MacFarlane, A. B. Chaplin, S. B. Hall, D. L. Officer, G. G. Wallace, and M. Forsyth, Polymer, 45, 1447 (2004)

26. D. J. Fermin, H. Teruel, and B. Schairfker, J. Electroanal. Chem., 401, 207 (1996).

27. J. C. Scott, J. L. Bredas, K. Yakushi, P. Pfluger, and G. B. Street, Synth. Met., 9, 165 (1984).

28. J. L. Bredas, J. C. Scott, K. Yakushi, and G. B. Street, Phys. Rev. B, 30, 1023 (1984).

29. M. Omastova, M. Trchova, J. Kovarova, and J. Stejskal, Synth. Met., 138, 447 (2003).

30. M. Bazzaoui, L. Martins, E. A. Bazzaoui, and J. I. Martins, Electrochim. Acta, 47, 2953 (2002)

31. W. Su and J. Iroh, Electrochim. Acta, 44, 2173 (1999).

32. A. Bozkurt, U. Akbulut, and L. Toppare, Synth. Met., 82, 41 (1996).

33. J. R. Macdonald, Impedance Spectroscopy, Wiley, New York (1987).

34. B. N. Grgur, N. V. Krstjic, M. V. Vojnovic, C. Lacnjevac, and Lj. Gajic-Krstajic, Prog. Org. Coat., 33, 1 (1998). 